\title{
Aménagement du territoire et urbanisme : le Québec a-t-il su relever le défi de la planification ?
}

\author{
Gérard Beaudet ${ }^{1}$ \\ Université de Montréal
}

\section{Introduction}

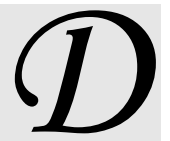
éjà, au début des années 1990, on pouvait se questionner sur l'importance et la pertinence des schémas d'aménagement relativement à l'évolution des établissements humains du sud du Québec. Certes, une décennie après le coup d'envoi de l'élaboration des premiers schémas d'aménagement et plus de 25 ans après le dépôt du rapport La Haye ${ }^{2}$, le Québec se donnait enfin les moyens d'une planification de l'urbanisation et de l'aménagement de son territoire humanisé. Qu'en était-il cependant du contenu de ces schémas et de leur potentiel d'encadrement et d'infléchissement des grandes tendances en matière d'utilisation et d'occupation du territoire? Si les schémas d'aménagement des MRC dans leur ensemble s'annonçaient de meilleure tenue que ceux qu'avaient adoptés les Communautés urbaines de Montréal, Québec et Hull, les résultats de ce vaste exercice étaient-ils pour autant à la hauteur des attentes trop longtemps contenues et des investissements consentis?

Bien qu'aucune véritable évaluation critique n'ait été effectuée, rares sont ceux qui auraient donné aux aménagistes et aux décideurs plus que la note de passage. Mais, suggérait-on, à défaut de révolutionner la planification physico-spatiale, l'exercice aurait au moins eu le mérite d'obliger les élus à se concerter en vue de s'entendre sur certaines préoccupations et sur certains objectifs communs. Mais dans l'ensemble, le bilan s'est avéré plutôt mince. La lecture des schémas ne suggérait-elle pas que le Québec était une société en plein développement, comme si la chute de la natalité, le vieillissement de la population, la désindustrialisation, l'exode des régions, l'effilochage croissant des lointaines périphéries métropolitaines, le vieillissement des infrastructures, les dommages causés à l'environnement, les coûts exorbitants de l'étalement urbain, l'érosion socio-économique de plusieurs quartiers anciens et de nombreuses petites villes étaient des phénomènes qui avaient épargné la Belle Province? En fait, on planifiait l'organisation du territoire comme on l'aurait fait à l'époque du dépôt du rapport La Haye, la sensibilité des auteurs du document Montréal Horizon $2000^{3}$ à certaines problématiques en moins.

Depuis, on s'est engagé dans la préparation et l'adoption de schémas d'aménagement de deuxième génération, cependant que les communautés métropolitaines ont entrepris l'élaboration de leurs schémas d'aménagement et de développement. Y a-t-il lieu de réviser l'appréciation précédente ? Malgré des disparités régionales certaines, le Québec se serait-il enfin donné les moyens d'une réelle cohérence aménagiste ? N'aurait-on pas plutôt, en imposant de nouveaux contenus à portée (en apparence ?) stratégique, relégué au second plan les préoccupations spécifiques à l'aménagement de l'établissement, occultant ainsi la nécessité d'un bilan? L'accent mis sur le paysage, la mise en valeur touristique des ressources ainsi que le développement durable ne témoignerait-il pas d'une fuite en avant caractérisée par la récupération de sensibilités émergentes, davantage que d'une certaine maturité ? Est-il même encore approprié de se préoccuper d'aménagement en ces temps où plusieurs, élus comme professionnels, ne jurent que par la gestion urbaine et territoriale?

En d'autres termes, le Québec aurait-il été en retard d'une révolution alors que le néolibéralisme et la 
mondialisation s'apprêtaient à saper les fondements idéologiques, technocratiques et financiers de l'État planificateur et interventionniste, dans un Québec où, au demeurant, l'essentiel des grandes interventions spatiales structurantes avaient été achevées en marge d'un véritable cadre de référence ? Un peu plus de vingt ans après l'adoption de la Loi sur l'aménagement et l'urbanisme, ne sont-ce pas là quelquesunes des questions qui méritent de retenir l'attention?

Par ailleurs, si la situation au Québec apparaît souvent pire qu'en de nombreux autres lieux où les traditions aménagistes sont plus et mieux enracinées, ce questionnement peut-il bénéficier d'une mise en perspective étendue ? La crise de l'urbanisme et de l'aménagement comme pratique professionnelle - crise au demeurant subordonnée à une crise de la planification -, la poursuite de tendances à l'étalement déplorées par plusieurs, le succès mitigé de solutions inspirées des mouvements du nouvel urbanisme et du Smart Growth, le développement de conurbations de villégiature, le dépeuplement des régions ressources et la restructuration de l'agriculture, l'accroissement des mobilités intramétropolitaines et le recul du transport public en regard de la part relative des déplacements, les ratés des formes traditionnelles de gouvernement métropolitain de même que la transformation radicale de l'offre commerciale constituent en effet quelques exemples de problèmes structurels qui ne sont pas l'apanage exclusif du Québec.

\section{Chronique de l'urbanisme et de l’aménagement du territoire au Québec}

Avec la Révolution tranquille, le Québec, fortement urbanisé depuis plusieurs décennies, entreprend une modernisation qui sera marquée par l'idée de progrès, une approche instrumentale du territoire et une rationalité gestionnaire dans les sphères d'activités de l'État. Traductions spécifiques de la planification, l'urbanisme et l'aménagement du territoire seront au programme de cette modernisation.

Jusque là, le territoire et son aménagement avaient été abordés en mode exclusivement sectoriel. Les politiques et programmes - par exemple en matière de colonisation, de voirie, de tourisme, de concession forestière - ne proposaient aucune vision intégrée de l'organisation et de l'évolution de l'établissement. Si quelques initiatives se démarquèrent par leur carac- tère ambitieux, par exemple en matière de régulation hydraulique des vastes bassins sur lesquels les droits d'exploitation hydroélectrique avaient été accordés à des monopoles, elles s'articulaient à des préoccupations essentiellement assujetties à des logiques d'entreprises, même là où des villes étaient créées à l'initiative de ces entreprises.

\section{La reprise de l'urbanisation au lendemain de la Seconde Guerre mondiale contribua à faire émerger des préoccupations à l'égard de certaines des conséquences des nouveaux modes d'organisation de l'espace.}

De ce point de vue, les travaux menés par Esdras Minville $^{4}$ à compter des années 1920 en vue de la réalisation d'inventaires systématiques des ressources naturelles du Québec, ainsi que l'expérience coopérative qu'il mena à Grande-vallée, son village natal de la péninsule gaspésienne, auront constitué des exceptions dans la mesure où le développement économique était mis au service d'une organisation rationnelle de l'établissement et au profit des populations.

À la ville, les premières réalisations en urbanisme s'inscrivaient, et ce depuis la fin $\mathrm{du}_{\mathrm{XIX}} \mathrm{e}^{\mathrm{e}}$ siècle, en filiation avec la pratique britannique. Cet héritage, incarné pour beaucoup par Thomas Adams (auteur entre autres du plan de la ville de Témiscamingue), sera réactivé dans les années 1950 avec la venue au pays de nombreux architectes et urbanistes britanniques. Ces derniers avaient été recrutés par la Société centrale d'hypothèques et de logements qui souhaitait ainsi sensibiliser les autorités municipales à l'importance d'adopter des plans et des règlements d'urbanisme et de promouvoir la pratique de la planification physico-spatiale. Cette approche s'est imposée presque sans partage.

La reprise de l'urbanisation au lendemain de la Seconde Guerre mondiale contribua par ailleurs à faire émerger des préoccupations à l'égard de certaines des conséquences des nouveaux modes d'organisation de l'espace. Accroissement des périmètres d'urbanisation, recul à l'avenant de l'agriculture et dilapidation des sols arables, empiétement sur les aires sensibles (milieux humides, boisés, etc.) et érosion des secteurs patrimoniaux commençaient en effet à inquiéter les 
planificateurs. C'est ainsi que l'urbaniste français Jacques Gréber fut mandaté au début des années 1950 par les villes de Montréal, de Québec et d'Ottawa pour proposer des mesures destinées à contenir l'urbanisation et à réorganiser les espaces hérités de la période industrielle. Ces propositions sont toutefois restées lettres mortes, sauf dans la région de la Capitale fédérale.

Malgré cette incursion hors des cadres habituels de l'urbanisme municipal, la région est demeurée le parent pauvre de l'urbanisme québécois. La dimension régionale a incidemment été davantage assumée par les géographes. À compter du début des années 1960 , la géographie descriptive, incarnée par l'œuvre de Raoul Blanchard ou par celle plus tardive de Roger Brière dans le champ du tourisme, a en effet cédé le devant de la scène à la géographie volontaire ou appliquée dont Michel Philipponneau et Philippe Pinchemel, professeurs invités au département de géographie de l'Université de Montréal au début de cette décennie, auront été les principaux porte-étendard. Les études régionales réalisées dans le cadre de la Loi ARDA (Loi sur l'aménagement régional et le développement agricole adoptée en 1961 par le gouvernement fédéral) seront incidemment un des principaux moments d'expérimentation de cette approche. Elles conduiront notamment à l'inventaire canadien des terres et au premier exercice de macro-zonage des potentiels d'utilisation des sols.

\section{Malgré cette incursion hors des cadres habituels de l'urbanisme municipal, la région est demeurée le parent pauvre de l'urbanisme québécois.}

L'importance croissante de la planification économique et la volonté d'atténuer les déséquilibres structurels régionaux contribueront toutefois rapidement à subordonner cet apport de la géographie appliquée à une démarche plus strictement économique. Au Québec, cette approche sera incarnée par le Conseil d'orientation économique du Québec, créé en 1961 et dont le mandat consistait à conseiller le gouvernement et à préparer un plan d'aménagement pour l'ensemble du Québec, puis par l'Office de planification et de développement du Québec (OPDQ), créé en 1968. Entre-temps, le Bureau d'aménagement de l'Est du Québec (BAEQ), mis sur pied en 1963, devait permettre au Québec d'expérimenter une démarche de planification régionale concertée influencée par l'expérience de la Tennessee Valley Authority menée presque trois décennies plus tôt dans le cadre du New Deal étasunien.

Durement éprouvée par des problèmes économiques qui n'avaient laissé aucun répit aux habitants de la région depuis les années 1920, la Gaspésie était toute désignée pour y mener une vaste opération de planification économique, sociale et territoriale. Si les travaux du Bureau d'aménagement de l'Est du Québec (BAEQ) n'ont pas donné les résultats escomptés, notamment en raison des dérives quant à l'avenir de certaines paroisses condamnées à la fermeture, l'expérience fut néanmoins et à des degrés divers une véritable école pour de nombreux professionnels.

Pendant ce temps, dans la région métropolitaine, les professionnels du service de l'urbanisme de la Ville de Montréal ébauchaient un document de planification voué à sensibiliser les élus aux problèmes et aux enjeux du développement du Grand Montréal. Présenté en 1967 à la Place des Arts, Montréal, horizon 2000 s'inspirait de l'approche française de la planification, et notamment du Cinquième Plan. La proposition s'articulait autour d'une conception hiérarchisée et polycentrée de l'établissement dont on estimait que la population s'élèverait à 6,7 millions d'habitants à la fin du siècle. Les grands axes autoroutiers soutenaient des poussées très organisées dont plusieurs aboutissaient aux villes satellites. Les interstices entre ces grands axes conservaient une vocation agricole ou étaient vouées à des affectations compatibles avec des préoccupations de conservation patrimoniale ou environnementale.

Malgré ces quelques incursions dans le champ des interventions à l'échelle régionale, que ce soit à Montréal ou ailleurs au Québec, l'urbanisme restera confiné, pour l'essentiel, aux espaces municipaux et aux interventions physico-spatiales.

Les premiers ratés de la planification technocratique à laquelle se rattachait ce courant étayé par les méthodes quantitatives se manifesteront toutefois très tôt. Alors que les populations de régions marginalisées par les restructurations économiques et celle des quartiers urbains voués à la quasi-démolition s'opposeront à une logique jugée inacceptable, parce que trop strictement instrumentale, on assistera à l'émergence 
de préoccupations environnementales et patrimoniales de mieux en mieux défendues à la faveur de mouvements populaires qu'encadreront l'advocacy planning, le militantisme communautaire et les groupes de ressources techniques.

L'Office de planification du Québec, créé en 1968 et rapidement devenu un des hauts lieux de la planification des interventions de l'État québécois, aura certes tenté d'intégrer à sa démarche certaines de ces préoccupations sociopolitiques. Une approche trop technocratique aura néanmoins empêché l'expression et la discussion des enjeux de la restructuration administrative en cours. Les résistances se multiplieront et la deuxième moitié des années 1970 sera celle des grands combats menés contre l'État et ses mandataires.

Le Québec ne fera évidemment pas bande à part dans la vague de contestations qui balaiera l'Occident à compter de mai 1968 et déferlera dans le champ urbain. La sociologie marxisante, notamment française, renouvellera de manière radicale la critique de la planification rationnelle initiée en Amérique du Nord par Jane Jacobs au début de la décennie. La mise sur pied de nombreux comités de citoyens dans les principales villes du Québec aura par ailleurs transposé sur le terrain cette critique. Les luttes urbaines et leur impact sur la pratique de l'urbanisme inscriront la dimension politique de l'urbanisme au cœur des préoccupations.

Entre-temps (1969), la création des Communautés urbaines de Montréal et de Québec et de la Communauté régionale de l'Outaouais avait fait naître certains espoirs en ce qui concerne l'évolution des trois principaux territoires métropolitains du Québec. Ces espoirs seraient toutefois vite et durablement déçus. Les limites inhérentes au statut politique de ces regroupements municipaux compromettront en effet irrémédiablement la mission qui leur avait été impartie par le gouvernement du Québec. La création du réseau des parcs-nature de la CUM reste un des rares héritages significatifs de cette période. L'expérience des Communautés urbaines et régionales aura en fait montré par l'absurde l'importance de la dimension politique de l'urbanisme.

La période qui s'étend de 1976 à 1990 fut caractérisée par la constitution d'un appareil législatif de plus en plus étendu et sophistiqué en matière d'urbanisme et d'aménagement du territoire. Certes, les pouvoirs des municipalités en matière d'urbanisme s'étaient accrus au tournant des années 1960 et 1970, et de nouvelles entités régionales avaient été constituées, dont les deux Communautés urbaines de Montréal et de Québec, la Communauté régionale de l'Outaouais et les Villes de Laval et de Mirabel. Ces regroupements donneront lieu à des démarches urbanistiques d'autant plus passionnantes pour ceux qui y étaient mêlés que l'urbanisme et l'aménagement demeuraient à l'époque des disciplines anémiques et peu valorisées en ces temps de forte croissance économique. Qu'il suffise de rappeler qu'à la veille de l'adoption de la Loi sur l'aménagement et l'urbanisme en 1979, seulement une centaine de municipalités parmi les quelque 1600 que comptait le Québec disposaient d'un plan d'urbanisme et que les réglementations en vigueur étaient habituellement des plus sommaires.

\section{Les luttes urbaines et leur impact sur la pratique de l'urbanisme inscriront la dimension politique de l'urbanisme au cœur des préoccupations.}

Le rapport Castonguay (Groupe de travail sur l'urbanisation), publié en 1976, soulignait incidemment la lenteur des progrès en matière d'urbanisme et d'aménagement du territoire. Cette lenteur était attribuée à un ensemble de causes, dont le peu d'empressement de l'État québécois à adopter une loi-cadre et à promulguer l'entrée en vigueur des schémas d'aménagement des deux Communautés urbaines, à l'éveil tardif des Québécois en regard des préoccupations urbanistiques, au caractère centralisateur et technocratique de la planification, à la rigidité des plans d'urbanisme et aux insuffisances des démarches et des méthodes de leur élaboration, ainsi qu'à la résistance de nombreux acteurs à toute forme de contrainte. Il faudra attendre encore trois ans avant qu'une loi sur l'urbanisme ne soit adoptée.

\section{La reconnaissance de la dimension politique de l'aménagement}

Revenons au cadre législatif en voie d'être constitué. Quelques dates repères méritent ici d'être soulignées : 1972, 1978 et 1985, modifications à la Loi sur les biens culturels; 1972 et 1978, adoption et amendement de la Loi sur la qualité de l'environnement; 
1974, adoption de la Loi sur les réserves écologiques; 1978, adoption de la Loi sur la protection des terres agricoles; 1979, adoption de la Loi sur l'aménagement et l'urbanisme. Un peu plus de dix années se seront donc écoulées entre le dépôt du rapport de la Commission provinciale d'urbanisme et l'adoption de la Loi sur l'aménagement et l'urbanisme, décennie particulièrement riche en événements, notamment les expériences du BAEQ, la contestation urbaine à Montréal, Québec, Hull, Trois-Rivières et Sherbrooke ainsi que le coup de force fédéral à Mirabel, qui auront montré le caractère éminemment politique de l'urbanisme et de l'aménagement du territoire.

La philosophie qui présidera à la formation des MRC et au libellé de la Loi sur l'aménagement et l'urbanisme témoignera avec éloquence de l'importance accordée à la dimension politique. À tel point que certains prétendront que cette loi définit davantage les modalités des relations entre les acteurs de l'urbanisme et de l'aménagement qu'elle ne propose un véritable cadre de référence aux pratiques et aux disciplines concernées. Elle ne permettait toutefois pas pour autant l'expression pleine et entière de cette dimension.

La reconduction de plusieurs des instruments déjà proposés dans le rapport La Haye ne contredit évidemment pas cette assertion. Les méthodes de l'urbanisme et de l'aménagement y demeurent en effet des plus conventionnelles, comme si rien n'avait véritablement évolué en ce domaine depuis les années 1950. Il faudra d'ailleurs attendre la deuxième moitié des années 1980 pour que soient introduits des mécanismes un tant soit peu innovateurs (notamment les règlements sur les Plans d'aménagement d'ensemble [PAE] et les Plans d'implantation et d'intégration architecturale [PIIA]).

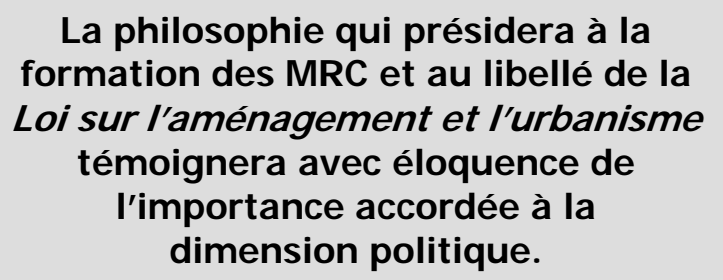

Cette reconnaissance, par la force des choses, du caractère politique de l'urbanisme et de l'aménagement aura beaucoup d'autres répercussions qui modifieront significativement la pratique de l'urbanisme.
L'explosion de la notion de patrimoine, que plusieurs associent à la destruction hautement symbolique de la maison Van Horne, en 1972, amènera le ministère des Affaires culturelles à intensifier ses interventions. Bon nombre d'organismes, dont Héritage Montréal et le Conseil des monuments et sites, fondés en 1976, veilleront d'ailleurs au grain et remporteront plusieurs batailles aux dépens de ceux qui perpétuaient les façons de faire des années 1960. À la même époque, en 1973, Hydro-Québec perdra la bataille de la JacquesCartier où l'on projetait la construction d'une centrale à réserve pompée. Ce premier échec retentissant conduira à la création d'une Vice-présidence de l'environnement et au développement d'une expertise exceptionnelle en matière d'évaluation environnementale et de consultation publique. Cela n'empêchera évidemment pas bon nombre d'autres contestations des projets de la société d'État, mais l'environnement aura désormais droit de cité.

Le rapport Castonguay avait par ailleurs mis en évidence les problèmes qui affectaient de plus en plus sévèrement les villes centres. Le rapport Legault (Groupe de travail sur l'habitation), publié la même année et portant spécifiquement sur l'habitation, confirmera le déclin démographique des villes centres et la détérioration des qualités du logement. Les programmes d'amélioration de quartier et de rénovation résidentielle, pilotés par la Société d'habitation du Québec et la Société canadienne d'hypothèques et de logements, constitueront une des réponses à ce problème qui pouvait de moins en moins être ignoré, voire banalisé. Ce regain d'intérêt pour la ville traditionnelle, la multiplication des luttes urbaines ainsi que l'émergence de préoccupations environnementales et patrimoniales auront de nombreuses répercussions. Ces mouvements créeront en quelque sorte la brèche dans les systèmes de gouvernance par laquelle seront explicitement introduites les préoccupations sociopolitiques, étroitement arrimées aux réflexions et pratiques françaises.

\section{Montréal, l'incontournable... et la mal aimée}

Cette période sera également marquée par un regain d'intérêt pour Montréal. En témoigneront : la publication, en 1977, de rapports de l'OPDQ et du MAM ${ }^{5}$; l'énoncé, en 1978, de l'Option préférable d'aménagement; la production, en 1984, de l'Option d'aména- 
gement de la région métropolitaine ${ }^{6}$; ainsi que le dépôt, en 1983, d'un rapport sur les parcs en milieu urbain ${ }^{7}$. Mais l'événement le plus significatif à l'égard de la métropole pourrait bien avoir été l'élaboration du projet Archipel. Vaste chantier inspiré des principes de la géographie volontaire, ce projet s'inscrivait dans le sillage de l'Option préférable de 1978. Cette proposition d'aménagement intégré de l'archipel pourrait, à certains égards, passer pour le baroud d'honneur d'un État-providence qui aurait reconnu, trop tard, l'importance de la métropole. Un examen plus approfondi montre toutefois qu'il s'est agi davantage d'un rendez-vous manqué qui contenait en germes l'essence d'une planification stratégique, opportuniste, à l'avant-scène de laquelle était placée la concertation des intervenants du milieu.

Cette ouverture montréaliste aura été de courte durée. Elle aura constitué un bref intermède dans un contentieux séculaire. Faut-il rappeler qu'en fin de la première moitié du XVII ${ }^{\mathrm{e}}$ siècle, les autorités de Québec souhaitaient empêcher Maisonneuve de réaliser son projet de fondation de Ville-Marie ? Ce contentieux pérenne a toutefois gagné en vigueur après la publication, en 1970, du rapport Higgins Raynauld-Martin qui recommandait de favoriser le développement de Montréal, posée comme la locomotive du Québec. Le Québec des régions, y compris celle de la capitale, ne l'entendrait toutefois pas ainsi. Lâchée par Ottawa au profit de Toronto à la même époque, Montréal devenait une métropole orpheline. Si on ne pouvait l'ignorer, on prendra bien soin, à Québec, de ménager ses arrières face à la grogne des régions. C'est ainsi qu'en 1997, au plus fort des discussions sur la réorganisation administrative de la région métropolitaine, Québec créait un ministère des Régions pour faire « contrepoids » à un ministère de la Métropole, au demeurant pas encore institué. Plus récemment, en janvier 2003, le ministre des Régions réclamait, pour les villes les plus importantes des territoires sous sa juridiction, des contrats de ville équivalents à celui que Québec entendait conclure avec la nouvelle Ville de Montréal.

Le Montréal de la fin des années 1980 sera aussi celui d'un autre chantier : le plan d'urbanisme et la révision systématique du cadre réglementaire. L'exercice aura été fascinant à bien des égards, en particulier pour toute une génération de jeunes professionnels confrontés à un défi de taille. La métropole rejoignait ainsi le peloton des MRC qui s'étaient dotées d'un

document proposant une vision aussi cohérente que possible, compte tenu de la nouveauté de la chose, du devenir de leur territoire. Ce plan connut toutefois un sort similaire à celui de plusieurs schémas d'aménagement et de non moins nombreux plans d'urbanisme: on se félicita de l'avoir adopté et on s'empressa de contrevenir à ses orientations, objectifs et énoncés.

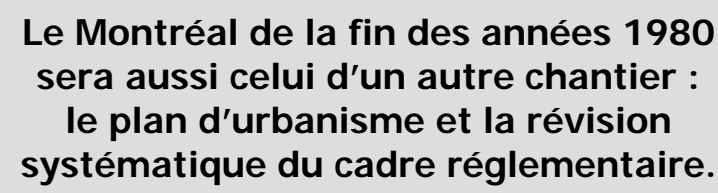

Les reculs des années 1990

Un peu moins de trente ans après le début des travaux de la Commission provinciale d'urbanisme, le Québec parvenait enfin à se doter d'une instrumentation minimale en matière d'urbanisme et d'aménagement du territoire, une aventure marquée par de grands espoirs, de multiples attentes, des avancées timides, des hésitations nombreuses et des reculs significatifs.

La période débutant grosso modo avec les années 1990 aurait pu être celle d'une consolidation des acquis. Toutes les MRC étaient dotées d'un schéma d'aménagement, la Ville de Montréal et la plupart des autres municipalités avaient adopté un plan et une réglementation d'urbanisme, de nouveaux pouvoirs avaient été conférés aux municipalités locales par le ministère des Affaires culturelles, le zonage agricole avait atténué certains des impacts les plus négatifs d'un étalement incontrôlé, les méthodes québécoises d'évaluation des impacts environnementaux et de consultation donnaient des résultats globalement satisfaisants, les urbanistes et les aménagistes étaient plus nombreux que jamais et on les retrouvait dans bon nombre de ministères, d'agences gouvernementales, de services municipaux d'urbanisme nouvellement créés et d'agences privées, de nouvelles pratiques avaient émergé, dont le programme Rues principales et les projets d'intendance privée pour la gestion de territoires sensibles.

Mais cette période en aura surtout été une de reculs sur de nombreux fronts. Invoquant la crise économique et les impératifs d'une rationalisation des dépenses, la classe politique prenait sa revanche. On s'épargnera la nomenclature des exemples de ces reculs. 
Certains pourraient être tentés d'invoquer la crise structurelle de l'urbanisme et des pratiques de l'aménagement pour les expliquer. Ce serait toutefois un peu court, d'autant plus que cette crise, alimentée par la crise de la planification, sévissait depuis au moins deux décennies. Si elle est bien réelle, elle ne saurait tout justifier. Le néolibéralisme et les détracteurs de l'État-providence y sont aussi pour quelque chose, et le Québec ne fait évidemment pas bande à part en ce domaine.

\section{Le Québec n'est jamais parvenu à se doter d'une véritable politique nationale d'aménagement du territoire et d'urbanisme.}

Mais se pourrait-il que les reculs aient été d'autant plus marqués ici que l'urbanisme et l'aménagement du territoire n'auraient jamais perdu, au cours des quarante dernières années, le statut de préoccupations secondaires ? C'est là une hypothèse qui paraît mériter examen. En d'autres termes, quarante ans d'efforts ne seraient pas parvenus à faire émerger une véritable culture urbanistique et aménagiste. Le rationalisme parfois un peu primaire, qui teinte encore fortement les approches et prête flanc à des critiques justifiées, est battu en brèche et laisse libre cours à une fuite en avant du côté du laisser-faire d'essence néolibérale, d'une planification stratégique et d'une gestion trop souvent indifférentes aux contenus, d'un formalisme de façade qu'incarne, à certains égards, le nouvel urbanisme, voire d'un incrémentalisme primaire.

Nous serions en fait dépositaires d'un héritage jamais véritablement pris en charge par la société québécoise en général et par la classe politique en particulier. Les débats des années 2000-2001 sur la réorganisation administrative des régions métropolitaines du Québec en ont témoigné avec une rare éloquence. Comme si la région de Montréal, tout comme celles de Québec, de Hull-Gatineau, de Sherbrooke ou de ChicoutimiSaguenay, n'était confrontée qu'à des problèmes de mauvaise gestion financière et d'iniquité fiscale. Sur ce terrain des réorganisations administratives municipales, tout suggérait que seule la dimension gestionnaire du problème méritait de retenir l'attention des décideurs. Or, faut-il le rappeler, l'urbanisme reste une des principales responsabilités des administrations municipales locales et régionales, une responsa- bilité qui a rarement été assumée de manière suffisamment satisfaisante pour qu'on puisse en faire abstraction lorsqu'on se lance dans de vastes opérations de fusion... ou de défusion.

Et pourtant, tellement reste à faire. Un peu partout, des initiatives montrent que l'urbanisme doit faire partie de la solution plutôt que d'être insidieusement associé à une contrainte. Le défi d'un repositionnement des questions d'urbanisme et d'aménagement comme enjeux de société, aussi considérable soit-il, doit conséquemment être relevé tant dans les officines ministérielles que dans les hôtels de ville et d'arrondissement ou sur le terrain.

\section{Conclusion}

Coïncidence ou convergence dont on n'a pas saisi la portée ? En 1976, l'année même de l'élection du Parti québécois, deux professeurs de l'Université de Montréal, Guy Dubreuil et Gilbert Tarrab, lançaient le premier ouvrage d'une collection vouée à l'exploration de «la manière de vivre » en ce presque Amérique $^{8}$. Replacée dans le contexte du mouvement d'affirmation identitaire qui mobilisait le Québec de l'époque, cette initiative pourrait sembler aller de soi. Malheureusement, elle n'aura pas de suite. La quête du pays mythique laissera dans l'ombre le pays réel. Comme si le Québec n'était guère plus que l'assise juridique de défense et d'illustration de la langue française et d'un modèle sociétal mis en place à la Révolution tranquille. Quant au pays réel, tout se passait comme s'il devait être soumis aux contraintes et aux impératifs d'un développement économique dont les retombées anticipées constitueraient la condition de possibilité d'un aménagement viable et socialement judicieux ${ }^{9}$.

Le Québec n'est jamais parvenu à se doter d'une véritable politique nationale d'aménagement du territoire et d'urbanisme. Faute d'un renouvellement des outils de la connaissance et de la compréhension de l'espace qui s'imposait dans les dernières décennies du siècle dernier, l'urbanisme et l'aménagement du territoire québécois sont restés enfermés dans une approche instrumentale et fonctionnelle de l'espace géographique. Ce faisant, leurs contenus ont trouvé peu d'écho dans la population. Celle-ci cherchera d'autres voies d'expression, dont celles extrêmement pernicieuses à terme, de la résistance et de la contestation. La 
réémergence de la dimension sensible de notre rapport à l'espace que révèlent les quêtes patrimoniales, environnementales et paysagères actuelles ne devraitelle pas, en la circonstance, nous inciter à relancer les réflexions en ce domaine? Après tout, il en va de l'avenir de la planification.

\section{Notes et références}

1 Gérard Beaudet est urbaniste, professeur titulaire et directeur de l'Institut d'urbanisme de la Faculté de l'aménagement de l'Université de Montréal. Il est également chercheur associé à la Chaire en paysage et environnement de la Faculté de l'aménagement.

2 La publication, en 1968, du rapport La Haye - du nom de son président - mettait fin aux travaux de la Commission provinciale d'urbanisme qui avaient été entrepris en 1963.

3 Étayé par de nombreuses études et cartographies thématiques, le document Montréal Horizon 2000 ne constituait pas à proprement parler un schéma d'aménagement ou un plan d'urbanisme. Il proposait plutôt une esquisse d'organisation territoriale optimale pour une agglomération de plus de 7 millions d'habitants.
4 Économiste et sociologue, cet intellectuel québécois de la première moitié $\mathrm{du} \mathrm{XX}^{\mathrm{e}}$ siècle inscrira ses réflexions et travaux dans le sillage du mouvement catholique, nationaliste et traditionaliste. Il sera directeur de l'École des Hautes Études commerciales de Montréal et membre des commissions Rowell-Sirois et Tremblay.

5 Direction générale de l'urbanisme et de l'aménagement du territoire, 1977.

6 Secrétariat à l'aménagement et à la décentralisation, 1984.

7 Comité interministériel sur les parcs en milieu urbain, 1983.

8 Dubreuil, Guy et Gilbert Tarrab (1976), Culture, territoire et aménagement, Éditions Georges Le Pape, coll. « Culture, territoire et aménagement $»$.

9 C'est là la thèse défendue par Gérard Beaudet, en collaboration avec Paul Lewis et avec des contributions de Jean Décarie et Daniel Gill (2000), dans Le pays réel sacrifié : la mise en tutelle de l'urbanisme au Québec, Québec, Nota Bene. 